\title{
Superficial Synthesis of CdS Quantum Dots for Efficient Perovskite- Sensitized Solar Cell
}

\author{
Rakesh K. Sonker, ${ }^{*, a, b}$ Rajkamal Shastri, ${ }^{c}$ Rahul Johari ${ }^{\mathrm{d}}$ \\ aDepartment of Physics and Astrophysics, University of Delhi, Delhi 110007, India \\ bDepartment of Physics, Acharya Narendra Dev College, University of Delhi, Delhi 110019, \\ India \\ cDepartment of Physics, Babasaheb Bhimrao Ambedkar University, Lucknow, Uttar Pradesh \\ 226025, India \\ ${ }^{\mathrm{d}}$ Department of Applied Sciences and Humanities, Jamia Millia Islamia, New Delhi 110025, \\ India \\ E-mail: rakesh.sonker81@gmail.com
}

\section{Supporting Information}

The structural characterization and crystalline phase analysis of CdS QDs were carried out by X-ray diffraction (Scheme 1). This also indicates that the lower reaction temperature helps the formation of hexagonal CdS QDs. The CdS QDs shows three broad characteristic peaks at $26.64^{\circ}, 43.95^{\circ}$ and $53.05^{\circ}$ correspond to the diffractions from the (111), (220) and (311) planes of CdS QDs standard card number JCPDS No.10-0191, respectively. The calculate of the size of hexagonal CdS QDs by Scherrer formula based on the full width at half maxima of diffraction peak

$$
D=\frac{0.9 \lambda}{\beta \cos \theta}
$$

where $\lambda$ is $\mathrm{X}$-ray wavelength, $\beta$ is the full width at half maximum intensity of the diffraction peak, $\theta$ is the Diffraction angle of the concerned diffraction peak in radians and D is the crystallite size in $\AA$. Parameters such as interplanar distance $(d)$, microstrain $(\varepsilon)$, dislocation density $(\delta)$ and distortion parameters $(g)$ are calculated using the following formulae given by 
Sonkawade et al. and Saxena et al. ${ }^{1,2}$

$d=\frac{\lambda}{2 \sin \theta}$

$\varepsilon=\frac{\beta \cos \theta}{4}$

$\delta=\frac{1}{D^{2}}$

$g=\frac{\beta}{\tan \theta}$

The physical constraints viz. $\mathrm{d}, \varepsilon, \delta$ and $g$ are calculated with regards to the prominent (111) peak $\left(2 \theta=26.64^{\circ}\right)$ of the CdS QDs as illustrated in Table 4.

Scheme 2 shows that the FTIR transmittance spectra of CdS QDs over $400-4000 \mathrm{~cm}^{-1}$. The FTIR spectroscopy was used to analyse the type of organic bonding adsorbed on the surface of the CdS QDs. Purified CdS QDs were put off in Milli-Q water and air-dried. The CdS QDs showed a characteristic band $\mathrm{O}-\mathrm{H}$ at $3105 \mathrm{~cm}^{-1}$. The peak at $2915 \mathrm{~cm}^{-1}$ was attributed to $\mathrm{CH}_{3}$ starching vibration, whereas Stretching of the CdS band can be seen at $653 \mathrm{~cm}^{-1}$. As the $\mathrm{CdS}$ QDs, bands at 1003, 1136, 1357 and $1589 \mathrm{~cm}^{-1}$ correspond to stretching of $\mathrm{C}-\mathrm{N}$, sulfide compounds, amide I bands of proteins and hydrogen bonding respectively.

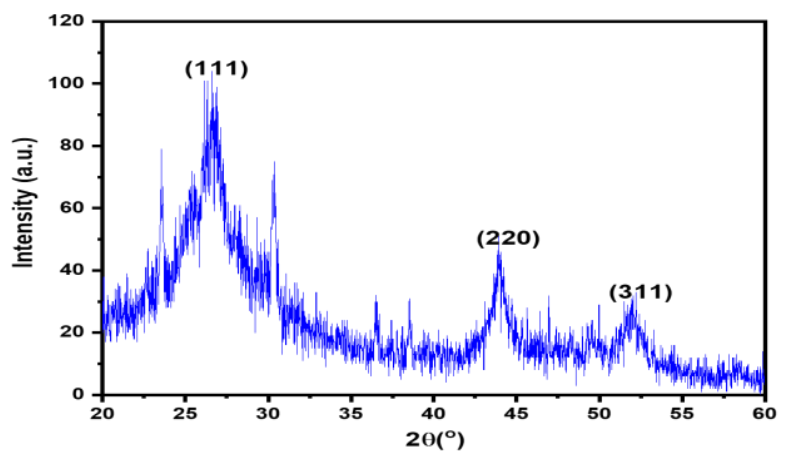

Scheme 1: XRD Pattern of Synthesised CdS QDs 


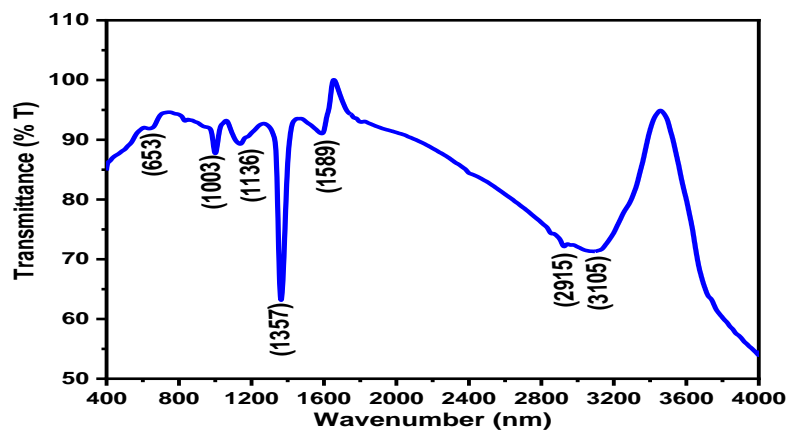

Scheme 2: FTIR Spectra of CdS QDs

Table 4: XRD Parameters of CdS QDs

\begin{tabular}{|c|c|c|c|c|c|c|c|}
\hline S. No. & $\mathbf{2 \theta}(\mathbf{d e g})$ & $\begin{array}{c}\text { FWHM } \\
(\mathbf{d e g})\end{array}$ & $\mathbf{D}(\AA)$ & $\mathbf{d}(\mathbf{\AA})$ & $\boldsymbol{\varepsilon}\left(\mathbf{x 1 0}^{-2}\right)$ & $\begin{array}{c}\boldsymbol{\delta}(\mathbf{x 1 0} \\
\mathbf{m}^{-2}\end{array}$ & $\mathbf{g} \%$ \\
\hline 1 & 26.64 & 2.4402 & 28.5 & 2.52 & 0.392 & 1.231 & 7.97 \\
\hline 2 & 43.95 & 1.7883 & 48.1 & 3.29 & 0.452 & 4.322 & 6.84 \\
\hline 3 & 53.05 & 1.6632 & 53.4 & 3.45 & 0.459 & 3.506 & 6.56 \\
\hline
\end{tabular}

\section{References:}

[1] M.R. Waikar, R.K. Sonker, S. Gupta, S.K. Chakarvarti, R.G. Sonkawade, Post- $\gamma-$ irradiation effects on structural, optical and morphological properties of chemical vapour deposited MWCNTs, Materials Science in Semiconductor Processing, 110 (2020) 104975.

[2] N. Saxena, T. Kalsi, P. Uttam, P. Kumar, Morphological evolution in nanocrystalline CdS thin films from flowers to salt rock like structures, Optical Materials, 84 (2018) 625-630. 